Ilona Renner* und Mechthild Paul

\title{
"Lotsensysteme“" zur Stärkung von Familien. Gelingensbedingungen für den Ausbau in Geburtskliniken
}

\section{"Pilotage services" to make families resilient. Key success factors for the implementation in maternity clinics}

https://doi.org/10.1515/pubhef-2021-0018

Zusammenfassung: Seit 2006 wurden in immer mehr Geburtskliniken „Lotsensysteme“ eingerichtet. Seither können Familien in akuten Belastungslagen frühzeitig in familienunterstützende Angebote der Frühen Hilfen übergeleitet werden. Im vorliegenden Beitrag wird anhand qualitativer Daten aus der Forschung des Nationalen Zentrums Frühe Hilfen der Frage nachgegangen, welche Erfolgsfaktoren die Einrichtung von „Lotsensystemen“ voranbringen und welche Hürden genommen werden müssen.

Schlüsselwörter: Entwicklung; Frühe Hilfen; Geburtsklinik; Gesundheit; Lotsensystem.

\begin{abstract}
Since 2006, more and more maternity clinics have set up "pilotage services". Since then, families in acute stress situations can be transferred from maternity clinics to family support services at an early stage. In this article, we use qualitative data from research conducted by the National Center for Early Intervention to examine the success factors that promote the establishment of "pilotage services" and the obstacles that need to be overcome.
\end{abstract}

Keywords: development; early childhood intervention; health; maternity clinic; pilotage services.

\section{Einleitung}

„Lotsensysteme“ sind ein Erfolgsfaktor im Gesamtsystem der Frühen Hilfen [1]. Seit 2006 haben sich immer mehr

\footnotetext{
*Korrespondenz: Ilona Renner, Nationales Zentrum Frühe Hilfen, Bundeszentrale für gesundheitliche Aufklärung, Maarweg 149 - 161, 50825 Köln, Germany, E-mail: ilona.renner@nzfh.de

Mechthild Paul: Nationales Zentrum Frühe Hilfen, Bundeszentrale für gesundheitliche Aufklärung, Köln, Germany
}

Kliniken auf den Weg gemacht und speziell ausgebildete Fachkräfte als „Lotsinnen“ in ihren Geburtsstationen eingesetzt. Seither können bei wahrgenommenen psychosozialen Belastungen die Lotsinnen persönlich Kontakt zu den Familien aufnehmen, sie zu einem Beratungsgespräch vor Ort einladen und ihnen - bei Bedarf - Zugang zum kommunalen Netzwerk Frühe Hilfen eröffnen [2]. Bezogen auf die Gesamtheit der im Detail unterschiedlich ausgerichteten Modelle in Deutschland stammen die bisher aktuellsten Daten zur Verbreitung aus dem Jahr 2017 [3]: Gut 30 Prozent der Kliniken mit mehr als 300 Geburten pro Jahr hatten bis zu diesem Zeitpunkt Lotsensysteme bereits aufgebaut bzw. den Aufbau fest geplant. Was hat die Kliniken dazu bewogen, diesen Schritt zu gehen? Und welche Hürden müssen Kliniken nehmen, die in Zukunft ein solches Lotsensystem aufbauen wollen?

\section{Hintergrund}

In den ersten Lebensjahren werden die Weichen für eine gesunde Entwicklung der Kinder gestellt [4]. Ein Teil der Eltern lebt jedoch in psychosozialen Belastungslagen, die eine gesundheits- und entwicklungsförderliche Versorgung und Erziehung ihrer Babys und Kleinkinder erschweren können. Ziel der Frühen Hilfen ist es deshalb, diese Eltern frühzeitig zu unterstützen, um einer potentiellen Beeinträchtigung der kindlichen Gesundheits- und Entwicklungschancen präventiv zu begegnen. Dabei stellt die oftmals schwierige Erreichbarkeit von Familien in Belastungslagen eine besondere Herausforderung dar.

Aus Forschung und Praxis ist bekannt, dass Angebote zur Unterstützung junger Eltern nicht alle Familien gleichermaßen erreichen, sondern dass sich in der Inanspruchnahme ein „sozialer Gradient“ zeigt: Oft sind es gerade Familien in belasteten Lebenssituationen, die 
- trotz objektiv hohem Präventionsbedarf - keine Kenntnis von Unterstützungsangeboten haben oder zögern, Angebote in Anspruch zu nehmen. Diese schwierige Erreichbarkeit ressourcenschwacher Familien wird auch als „Präventionsdilemma“ [5] bezeichnet. Zur Überwindung dieses Präventionsdilemmas ist es von zentraler Bedeutung, geeignete „Lotsensysteme“ aufzubauen.

Geburtskliniken gehören dabei zu den wichtigsten Kooperationspartnern der Frühen Hilfen. 98 \% der Kinder kamen 2016 in Geburtskliniken zur Welt [6]. Ärztinnen und Ärzte sowie das Pflegepersonal genießen ein ausgesprochen hohes Vertrauen bei (werdenden) Müttern. Eltern sind zudem in der Zeit rund um die Geburt, in der sich die Familie neu gestaltet, besonders offen für Beratungs- und Unterstützungsangebote.

\section{Methode}

Um Gelingensbedingungen und potentielle Hürden beim Aufbau von „Lotsendiensten“ in Geburtskliniken $\mathrm{zu}$ ermitteln, hat das Nationale Zentrum Frühe Hilfen das Deutsche Krankenhausinstitut (DKI) mit der Durchführung einer quantitativen und qualitativen Teilstudie des ZuFa (Zusammen für Familien) Monitorings [7] beauftragt. An der quantitativen Repräsentativbefragungvon 2017 nahmen 383 Geburtskliniken mit mehr als 300 Geburten pro Jahr teil (57\%).

Der qualitative Studienteil bestand aus elf Telefoninterviews mit Befragten aus Geburtskliniken, die zwar noch keine oder nur wenige Aspekte eines „Lotsensystems“ umgesetzt hatten, aber dennoch Bedarf für eine verbesserte Versorgung ihrer psychosozial belasteten Wöchnerinnen sehen. Zusätzlich wurden drei Workshops mit Krankenhäusern, die sich bereits auf den Weg in Richtung Lotsensystem gemacht haben, durchgeführt, dokumentiert und ausgewertet [8].

\section{Erfolgsbedingungen}

Ob „Lotsensysteme“ in Geburtskliniken eingeführt werden, hängt stark von der Initiative einzelner Mitarbeitender des Krankenhauses oder der kommunalen Frühen Hilfen ab. So haben sich in einem Teil der Kliniken, die an den telefonischen Interviews teilgenommen haben, „Frauen von den Frühen Hilfen [...] vorgestellt“, „die haben uns angefragt und wir haben uns getroffen und haben es gut befunden“ [8]. Die Idee eines „Lotsensystems“" hat in den letzten Jahren viel Anklang gefunden.
Dies liegt auch daran, dass in der Geburtshilfe ein stetig zunehmender Bedarf an familienunterstützenden Angeboten nach der Geburt wahrgenommen wird: So geben in dem quantitativen Studienteil 67\% der Befragten an, dass der Anteil psychosozial belasteter Mütter in den letzten Jahren zugenommen hat [7].

Während der erste Anstoß zur Implementierung eines Lotsensystems von einzelnen Mitarbeitenden des Krankenhauses oder den kommunalen Frühen Hilfen erfolgt, braucht es im weiteren Prozess sog. „Kümmerer“: Die Studienergebnisse zeigen, dass insbesondere Führungskräfte, die das Angebot aktiv unterstützen, ein entscheidender Erfolgsfaktor sind [8]. Gelingt es, die Führungskräfte zu überzeugen, dann sind in einem zweiten Schritt für die weitere Implementierung von „Lotsensystemen“ engagierte Mitarbeitende von Bedeutung, insbesondere Ärztinnen bzw. Ärzte und Pflegekräfte. Eine erfolgreiche Umsetzung verlangt zudem eine Klärung der Zuständigkeiten, transparente Kommunikation und eine besondere Offenheit für interdisziplinäre bzw. -professionelle Zusammenarbeit. Nicht zuletzt benötigt der Prozess finanzielle Ressourcen: Eine (Anschub-) Finanzierung wird als wesentlicher Erfolgsfaktor genannt. Lotsendienste können grundsätzlich sowohl im Rahmen der Bundesstiftung Frühe Hilfen als auch aus Landesmitteln gefördert werden, wenn diese nicht anderweitig gebunden sind.

\section{Herausforderungen}

Mehrere Interviewpartner berichteten, dass die Frühen Hilfen bzw. psychosoziale Belastungen von Familien nicht zum Kerngeschäft ihres Krankenhauses bzw. der ärztlichen Versorgung zählen. Dies spiegelt sich exemplarisch in dem folgenden Zitat wider: „[V]iele Kollegen aus der Ärzteschaft wollen damit nichts $\mathrm{zu}$ tun haben, betrachten es nicht als ihre Aufgabe, sich um solche Probleme zu kümmern, [...] da werden medizinische Innovationen mehr anerkannt“ [8]. Es wird angemerkt, dass „Kliniken [...] da mehr als Wirtschaftsunternehmen geführt [werden], da passt das nicht rein, schwarze Null zählt, sind für die eher Nebensächlichkeiten“, und dass Prävention sowie auch Nachsorge nicht die primäre Aufgabe der Krankenhäuser seien.

Genannt wurden auch Barrieren, die Probleme bei der Finanzierung sowie das mangelnde Verständnis für die Belange der Frühen Hilfen bzw. für die poststationäre Versorgung betreffen [8]. Erschwerend bei der Implementierung von „Lotsensystemen“ wirkte sich auch die 
Notwendigkeit aus, sich mit diesen neuen Herausforderungen auseinanderzusetzen, um Zuständigkeiten und Kompetenzbereiche zu klären [8]. Ebenso wurde die zeitund personalintensive Versorgung von (psychosozial) belasteten Frauen angeführt, die in deutlichem Kontrast zu den knappen zeitlichen und personellen Ressourcen im Krankenhaus steht [8]. Interessant ist dabei, dass jedoch gerade dieser Aspekt ein Grund dafür ist, dass Mitarbeitende in Kliniken, die bereits „Lotsensysteme“ implementiert haben, zufrieden mit dieser Innovation sind: Die Einbindung von (zusätzlichen) Frühe HilfenFachkräften, die speziell für die Versorgung psychosozial belasteter Wöchnerinnen ausgebildet sind, wird als Entlastung für Ärztinnen bzw. Ärzte und Pflegende begriffen und durchweg sehr positiv bewertet [3].

\section{Fazit}

Frühzeitige psychosoziale Unterstützung für Eltern mit Hilfebedarf kann zu einer verbesserten Chancengerechtigkeit der Kinder beitragen. Dazu sollten Eltern möglichst bereits rund um die Geburt für eine (spätere) Hilfeannahme gewonnen werden. In Geburtskliniken gibt es heute schon vielfach bewährte und erprobte Ansätze zur Ansprache, Beratung und Vermittlung von Familien, sogenannte „Lotsensysteme“. Sind sie eingerichtet, werden „Lotsensysteme“ vom Klinikpersonal als Entlastung erlebt. Die Kenntnis von Erfolgsfaktoren sowie Herausforderungen, wie beispielsweise Fragen der Finanzierung oder der Ansprache von Klinikleitung und -personal, kann dazu beitragen, dass diese „Lotsensysteme“ - zum Wohle der Kinder und ihrer Familien - in Zukunft weiter ausgebaut und einem stetigen Qualitätsentwicklungsprozess in den Klinikabläufen unterzogen werden.

\section{Autorenerklärung}

Autorenbeteiligung: Alle Autoren tragen Verantwortung für den gesamten Inhalt dieses Artikels und haben der Einreichung des Manuskripts zugestimmt. Finanzierung: Das Projekt wurde gefördert aus Mitteln der Bundesstiftung Frühe Hilfen des Bundesministeriums für Familie, Senioren, Frauen und Jugend. Interessenkonflikt: Die Autoren erklären, dass kein wirtschaftlicher oder persönlicher Interessenkonflikt vorliegt. Ethisches Statement: In Übereinstimmung mit der Deklaration von Helsinki wurden die Teilnehmer über das genaue Verfahren der
Studie informiert und haben freiwillig teilgenommen. Alle Teilnehmer gaben ihre Zustimmung. Die Studie wurde von der Ethikkommission „freiburger ethik-kommission“ zugelassen (feki-Code: 016/1868).

\section{Author Declartion}

Author contributions: All authors have accepted responsibility for the entire content of this submitted manuscript and approved submission. Funding: The project received funding by the Federal Foundation for Early Childhood Intervention of the Federal Ministry for Family Affairs, Senior Citizens, Women and Youth. Conflict of interest: Authors state no conflict of interest. Ethical statement: In accordance with the Helsinki Declaration, the participants were informed about the exact procedure of the study and took part voluntarily. All participants gave their approval. The study was approved by the Ethics Commission "freiburger ethic-commission" (feki-code: 016/1868).

\section{Literatur}

1. Renner I, Saint V, Neumann A, Ukhova D, Horstmann S, Böttinger $U$, et al. Improving psychosocial services for vulnerable families with young children: strengthening links between health and social services in Germany. Br Med J 2018;363:k4786.

2. Schmenger S, Schmutz E, Backes J, Scharmanski S. Zentrale Qualitätskriterien für Lotsendienste der Frühen Hilfen in Geburtskliniken. Fachliche Anforderungen für die weitere Profilierung. Eckpunktepapier. Nationalen Zentrum Frühe Hilfen (NZFH), Herausgeber, Köln, 2020.

3. Scharmanski S, Renner I. Geburtskliniken und Frühe Hilfen: Eine Win-Win-Situation? Ergebnisse aus dem NZFH-Forschungszyklus „Zusammen für Familien“ (ZuFa-Monitoring), Nationalen Zentrum Frühe Hilfen (NZFH), Herausgeber, Köln, 2019. https://doi. org/10.17623/NZFH:K-GebKliZuFa.

4. Hughes K, Bellis MA, Hard-Castle KA. The effect of multiple adverse childhood experiences on health: a systematic review and meta-analysis. Lancet Public Health 2017;2:e356-66.

5. Bauer U. Das Präventionsdilemma. Potenziale schulischer Kompetenzförderung im Spiegel sozialer Polarisierung. Wiesbaden: VS, 2005.

6. Eigene Berechnung des NZFH, basierend auf: Gesundheitsberichterstattung des Bundes, 2018. www.gbe-bund.de (23.7.2019).

7. Renner I, Scharmanski S, van Staa J, Neumann A, Paul M. Gesundheit und Frühe Hilfen: Die intersektorale Kooperation im Blick der Forschung. Bundesgesundheitsbl 2018;61:1225-35.

8. Steffen P, Blum K. Zusammen für Familien. Das ZuFa Monitoring Geburtsklinik Forschungsbericht1: Telefoninterviews und Workshops, 2018. https://www.dki.de/sites/default/files/2019-05/ Geburtskliniken\%20in\%20den\%20fruehen\%20Hilfen.pdf. 